\title{
The Integration of the Rehabilitation of Psychiatric Patients into the Primary Health
}

Care System

Prof N.E. SOKHELA Phd

Deptartment of Nursing Science Unitra

Prof L. R. UYS D.Soc.

Department of Nursing University of Natal

\section{abstract}

This study done in rural and semi-urban clinics examined the ability of primary health care nurses in providing rehabilitation of psychiatric patients in the Primary Health Care service. The objectives of the study were to train and evaluate registered nurses' ability to implement rehabilitation to psychiatric patients in the community.

Registered nurses were trained over a period of 10 days. Each client who visited the clinic had a rehabilitation plan drawn with the client and family. Families participated in the training of clients while nurses were trained to identify target symptoms, draw a plan to be followed by the client and his family, set rehabilitation goals and the steps to achieve the goals. The project was implemented over a period of 12 months. Records were then reviewed 1 year after implementation and at 18 months to determine the performance of nurses. Evaluation was done per clinic. Each clinic was evaluated and differences were found and where performance was poor, re-education was done. Each clinic was seen as a case.

A record review was done to determine the level of rehabilitation based on the following:

- $\quad$ identification of target symptoms

- plan for the patient

- plan for the family

- $\quad$ setting of rehabilitation goals

- steps to achieve goals

- level of vocational rehabilitation

\section{1. introduction}

Rehabilitation of psychiatric patients is a necessary component of the psychiatric services that supports the client and family during the period of recovery. Munich, (1993) describes rehabilitation as a biopsychosocial approach that assumes that all people have a capacity to learn, compensate and adapt but needs support from others to enable the client to exercise maximum autonomy and self direction. Anthony et al (1990) reports that rehabilitation is a bridge created after the health authorities realised that psychiatric hospitals were not therapeutic and that simply moving clients into the community would not bring in improvement in their quality of life.

Rehabilitation has basic elements which act as a guide to planning. Difficulties exist in the implementation of rehabilitation in South Africa due to the absence of a clear policy. This has led to segregation of rehabilitation from the rest of the health care.

The problem addressed in this article is how psychiatric rehabilitation can be integrated into the PHC services offered in government clinics by PHC nurses. More specifically questions addressed were:

1. What is the quality of rehabilitation diagnosis and rehabilitation plans given by the PHC nurse?

2. What is the level of vocational rehabilitation for each clinic ?

\section{terms used}

\section{Rehabilitation Plan}

A series of proposals of activities decided by the client, family and health provider for the rehabilitation of the client aimed at the achievement of set goals.

\section{Vocational Rehabilitation Level}

This is the level of functioning possessed by the client at a specific time, and refers to all self-care activities as well as working around the house and external job activities.

\section{Rehabilitation Diagnosis}

It is a statement which explains the present existing skills of the client, the 
needed skills and support which will enable the client to live and or work in a specific environment.

\section{2. literature survey}

Rehabilitation is an approach in which clients take an active part in the provision of health care aimed at the prevention of relapse. The effect of mental illness has an impact on social skills, vocational skills and coping skills and therefore restrict the client from performing in the way regarded as normal by society. The affected areas in the clients functioning are a focus for rehabilitation (Uys, 1991). Rehabilitation should also include the family who care for the client in the absence of the health professional. Families need to be educated on the nature, course and the effects of the illness on the day to day functioning of the affected person. If the client is on medication it is also important to educate the family on the effects of medication and relapse prevention (Sullivan, 1994)

Munich (1993) remarks that all people have a capacity to learn and adapt and that disabled clients should be given the chance to exercise maximum autonomy and self direction. He further puts emphasis on the fact that rehabilitation approach focuses on training clients on interpersonal, cognitive and independent living skills. The goals of psychiatric rehabilitation include sustaining the improvement of symptoms through medication, family, psycho-education, helping the client to achieve access to resources (Munich, 1993).

In order for rehabilitation to be planned and the patient's capacities are assessed and the remaining capacities are enhanced. Rehabilitation has the following basic elements described by Uys (1991):

\section{Increasing skills}

Skills are increased to enable the client to cope better with the stress and in some cases prevent stress. The skills are those affected by the illness such as vocational, life and social skills and any other skills needed by the client or his/her family.

\section{Increasing support}

This is any action that increase the support received by the client and family and assist them in preventing breakdown and promote health in the form of material assistance and psychosocial support.

\section{Manipulating resources}

This aspect includes marketing the service to the client or marketing the client to the service. It may also include negotiation for changes within the service to make the service appropriate for the client or advocate for the improvement or creation of a service.

4. Optimilizing symptoms control Psychiatric patients remain with troublesome symptoms during their life. Successful rehabilitation depends on the control of symptoms through medication and at times psychotherapy or both. For the client to be sustained at home and at work the rehabilitation worker must determine which symptoms need to be controlled, as symptom control that is adequate to enable the client to live within the family may not be adequate for work. The target symptoms are therefore the focus of rehabilitation.

Clients are taught to recognise symptoms and predictive signs of relapse and to take an action to counteract them. Family members should also be able to identify the first symptoms that appear as an indication of relapse and act immediately.

\section{General public education}

Successful integration of the client within the family and society depends on the attitudes of such families and societies. Steps should be taken to change attitudes purposefully and should be linked to increasing the support for the family and the community.

Like all other patients, psychiatric patients have needs that have to be met. Kraus \& Slavinsky (1982) list the following needs:

- $\quad$ a need to be taken care of

a need for social interaction a need for relief from psychiatric symptoms

a need for basic life necessities a need for hope

the needs for others

Psychosocial rehabilitation enables clients to meet these needs.

In South Africa psychiatric care is still in its de-institutional era and rehabilitation has not yet happened in the manner expected as described by Anthony, Cohen \& Farkas (1990). Although rehabilitation had been included earlier, its provision was not seen as part of health care with some components under the welfare services. For the success of rehabilitation there should be a close cooperation between health, welfare, labour and the communities so that the services of these sectors are integrated within a PHC clinic.

The National Health Policy discusses rehabilitation as a component of the National Health Department but makes no provision at Provincial level. At district level, the Act provides for provision, maintenance and co-ordination of community based rehabilitation centres. The decision on whether such centres depends on districts or province has to be made. If these centres fall under the district and are far from other communities, the objective of accessible health care may not be met.

Rehabilitation in South Africa rests in the hands of nurses especially those in the $\mathrm{PHC}$ setting. These nurses have not been prepared for the provision of this service. A need therefore arises for the PHC nurses to be trained to handle chronic mental illness within their clinics.

\section{3. research methodology}

A case study design was used in which each clinic was seen as a case using both qualitative and quantitative data. The method involved an intensive study of the performance of nurses and the problems encountered in each clinic.

\section{educational programme}

The programme used to teach PHC consisted of :

- a skill based approach with only theory necessary to master the skills.

methodologies which sought to promote attitude change.

The programme started with a ten day workshop followed by 1 year of implementation and evaluation and a second evaluation after 18 months. Students were provided with compiled books in which there were objectives to be achieved for each unit and the literature to give information. These books were designed in line with the training manuals developed by WHO used for the training of health professionals in Columbia and by $\mathrm{PHC}$ nurses in Zimbabwe (Reeler, 1987).

\section{Teaching Methods}

Lessons were conducted in the form of workshops followed by group discussions of 3-5 students in a group.

\section{Evaluation Methods}

Continuous evaluation was done and two tests were given during the 10 day period but no definite marks were allocated. Discussions were centred round the identified weak areas. Case histories were given and participants required to make a rehabilitation diagnosis. Role plays of given case histories enabled the participants to play different roles in determining what needed to be included in the rehabilitation plan.

\section{sampling for case studies}

Six clinics were purposively selected to form the sample because they were within the supervision distance and also represented urban and rural clinics. At least $50 \%$ of registered nurses in each 
clinic were trained to be able to provide rehabilitation to psychiatric patients within their clinics and homes.

\section{sample}

A total of 42 files from the six clinics formed the sample. The files from each clinic were purposively selected from files of clients who visited the clinic regularly for rehabilitation and review. A number of between 4 to 12 files was selected depending on the available clients for rehabilitation. Some clinics had more files while others had less. Most clients were not consistent in visiting the clinics due to the problem of absence of medication supplies. Instead they visited the outpatient clinic where medication was available. These clients could not be included in the sample.

\section{data collection}

Data was collected at 1 year after implementation and at 18 months. The data collection instrument accompanied each file and sent to two independent evaluators so that each file was evaluated on the same aspects by each evaluator independently. The independent evaluators were both psychiatric nurse specialists.

\section{ethical consideration}

Written permission was obtained to conduct the research and to use the files from the Department of Health in Bisho, Department of Health Umtata, Umtata General Hospital and Umtata Municipality. Although client files were used, code names were used for each file for
PHC nurses and their supervisor. They had no previous exposure to such activities as goal setting and working out plans for a client and family to follow. Much support and teaching was necessary.

\section{1 identification of target symptoms}

Participants were able to identify the first indicative symploms with the client and family. The family and client were educated to observe for the appearance of the symptoms as an indication of relapse. These symptoms were the target on which the rehabilitation plans were centred. The results shown in figure 1 reflect the following:-

\section{instrument}

In order to measure the registered nurse's performance in rehabilitation planning and implementation, a rating scale with 6 closed ended questions was designed by Uys (1996). These questions evaluated the following aspects:

- $\quad$ Ability of participants to identify target symptoms. Clarity and appropriateness of the plan for the patient and for the family. Rehabilitation goals. Appropriateness of the steps to achieve goals. The level of rehabilitation for each case.

\section{content validity}

The content of the instrument covered all major aspects of rehabilitation. The instrument was designed based on the extensive information from literature. No reliability testing was done. Table 3.1 shows the content that form focus of the evaluation.

\section{Table 3.1 Areas covered by content on rehabilitation}

\begin{tabular}{|l|}
\hline Areas covered in the programme \\
\hline 1. Rehabilitation Diagnosis \\
\hline 2. Rehabilitation Plans \\
\hline 3. Skills teaching \\
\hline 4. Relapse prevention \\
\hline 5. Vocational rehabilitation \\
\hline 6. Psycho-education \\
\hline
\end{tabular}

\section{Figure 1 : Identification of target symptoms per clinic (Full \& Partial)}

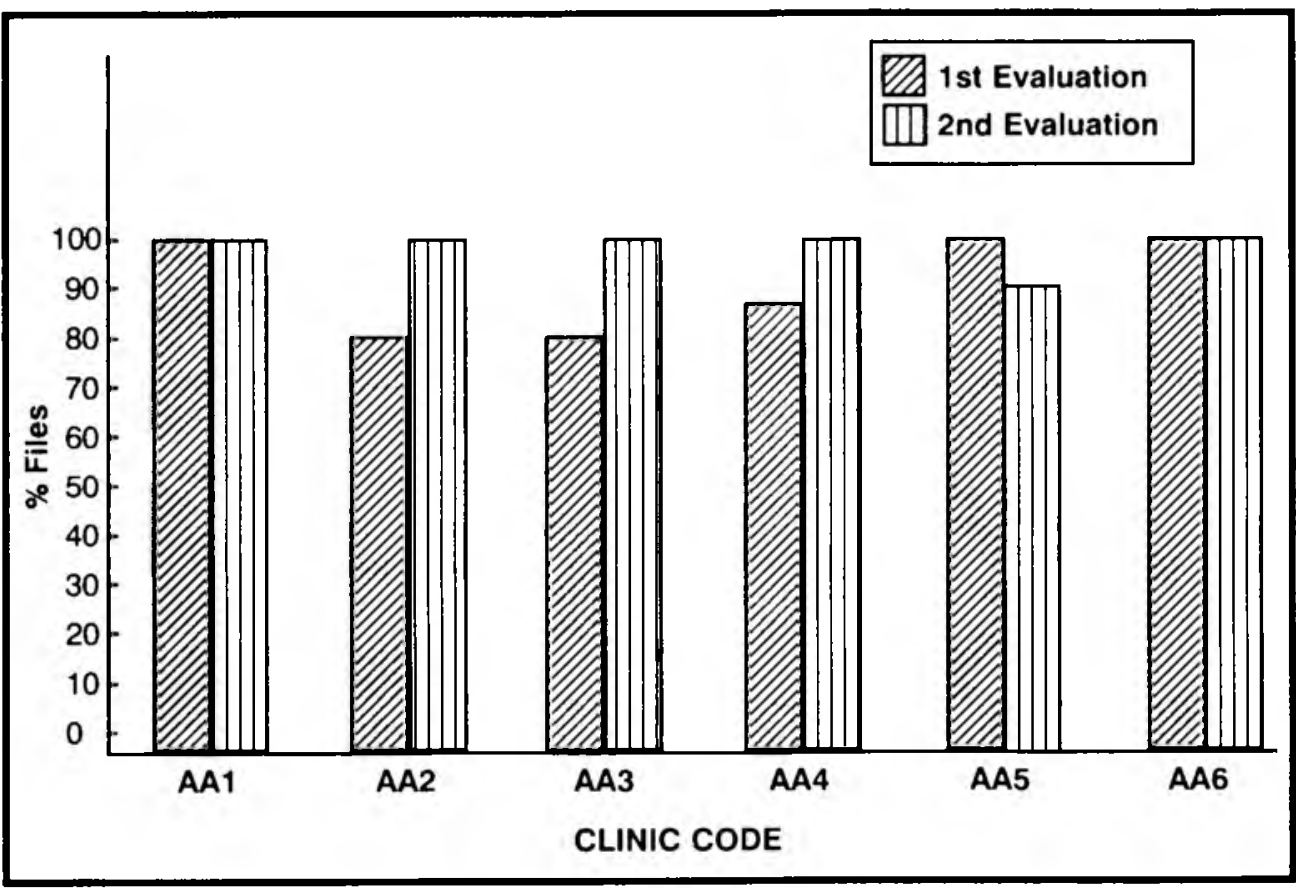

confidentiality. Clinic committees gave permission for implementation of the project in their settings. To maintain anonymity names of participants and clinics used in the study will not be mentioned. Anonymity in responses was maintained as there were no names in all instruments used for evaluation. Individual permission was obtained from each client or family member, by registered nurses participating in each clinic, based on the full explanation given to the patient/family about the nature of the study.

\section{4. results}

The implementation of the rehabilitation counselling process was not easy for
First Evaluation (one year after implementation)

Clinic AA1 could identify target symptoms in $100 \%$ of the files.

Clinic AA2 could identify $80 \%$ of the target symptoms.

Clinic AA3 could identify $80 \%$ of the target symptoms.

Clinic AA4 could identify $88 \%$ of the target symptoms.

Clinic AA5 could identity $100 \%$ of the target symptoms.

Clinic AA6 could identity $100 \%$ of the target symptoms.

Partial identification of symptoms is also a positive result although a very minor aspect of the target symptom was left out. The percentage of the partially identified target symptoms if added to the above 
results increases the nurses' ability in the performance of the skill.

Unidentified target symptoms were the concern for retraining in clinic AA2, AA3, and AA4 although the percentage ranged between 12 and $20 \%$.

Second Evaluation (after 18 months)

In the Second evaluation following retraining of the three clinics which could not identify all target symptoms the results were $100 \%$ in all the clinics.

\section{2 plan for the patient on target}

\section{symptoms}

The plans set were not clear and did not fully target the symptoms presented. With more exposure and exercises participants developed a better plan to assist the patient. The plans involved steps to be taken if the symptoms appear at night or at work. The results shown in figure 2 reflect the following :

Clinic AA1 could set appropriate plans in $90 \%$ of the files after 12 months and in $100 \%$ of the files after 18 months.

Clinic AA2 could set appropriate plans in $70 \%$ of the files after 12 months and $100 \%$ of the files after 18 months.

Clinic AA3 could set appropriate plans in $65 \%$ of the files after 12 months and in $100 \%$ of the files after 18 months.

Clinic AA4 could set appropriate plans

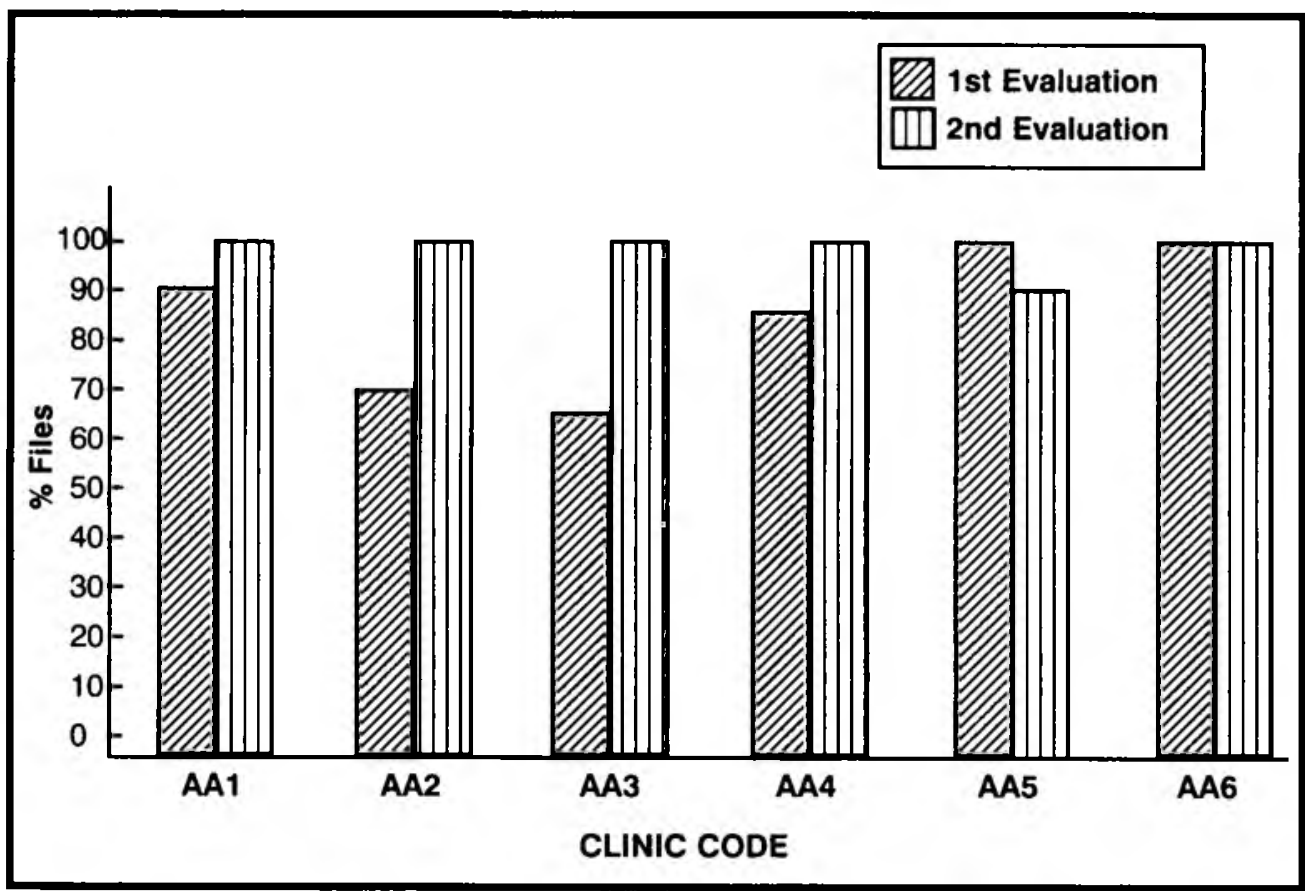

in $88 \%$ of the files after 12 months and $100 \%$ after 18 months.

Clinic AA5 could set appropriate plans in $100 \%$ of the files but decreased to $90 \%$ after 18 months.

Clinic AA6 could set appropriate plans in $100 \%$ of the files in both the first and second evaluation.

\section{3 plan for the family on target symptoms}

The plans set had to assist the family in terms of developing the ability of the family to cope with the illness of the patient. These plans dealt with handling conflicts, concerns and expectations of the family. The evaluation shows that:-

Clinic AA1 could set appropriate plans in $90 \%$ of the files in the first evaluation and $100 \%$ in the second evaluation.

Clinic AA2 could set appropriate plans in $70 \%$ of the files in the first evaluation and $100 \%$ in the second evaluation.

Clinic AA3 could set appropriate plans in $80 \%$ of the files in the first evaluation and $100 \%$ in the second evaluation. Clinic AA4 could set appropriate plans in $80 \%$ of the files in the first evaluation and $88 \%$ in the second evaluation.

Clinic AA5 could set appropriate plans in $100 \%$ of the files in both first and second evaluation.

Clinic AA6 could set appropriate plans in $70 \%$ of the files in the first evaluation and $100 \%$ in the second evaluation.

\section{4 setting of rehabilitation goals}

These goals were determined on the basis of what the client wanted to achieve and what the relatives wanted the patient to achieve. The goals were aimed at assisting the client to be able to function within the environment in which he/she lives. Goal setting improved dramatically during the course of training as a result of exercises given on goal setting. For most of the 
clients initial goals dealt with functioning in the home setting. There was a small group of patients who were working and in these cases supervisors were involved in setting rehabilitation goals.

\section{5 steps to achieve the set goals}

Again, nurses showed a dramatic improvement in their ability to make realistic plans to achieve goals. The activities usually involved the family teaching the patient to do something and then monitoring execution of the actions. The results in figure 4 reflect the following:

Clinic AA1 had achieved correct steps in $80 \%$ of the files in the first evaluation and in $100 \%$ of the files in the second evaluation. Clinic AA2 had correct steps in $100 \%$ of the files in both first and second evaluation.

Clinic AA3 had correct steps in $80 \%$ of the files in the first evaluation and in $100 \%$ of the files in the second evaluation.

Clinic AA4 had correct steps in $88 \%$ of the files in the first evaluation and in $100 \%$ of the files in the second evaluation.

Clinic AA5 had correct steps in $100 \%$ of the files in both first and second evaluation.

Clinic AA6 had correct steps in $70 \%$ of the files in the first evaluation and $90 \%$ of the files in the second evaluation.

\section{6 level of vocational rehabilitation}

For each client the level of rehabilitation was calculated. Scores of patients were added for each clinic and reflected as the level of rehabilitation achieved by that clinic. For each client level of functioning at the beginning was rated and given a

\section{Figure 5 : Level of Vocational Rehabilitation per clínic}

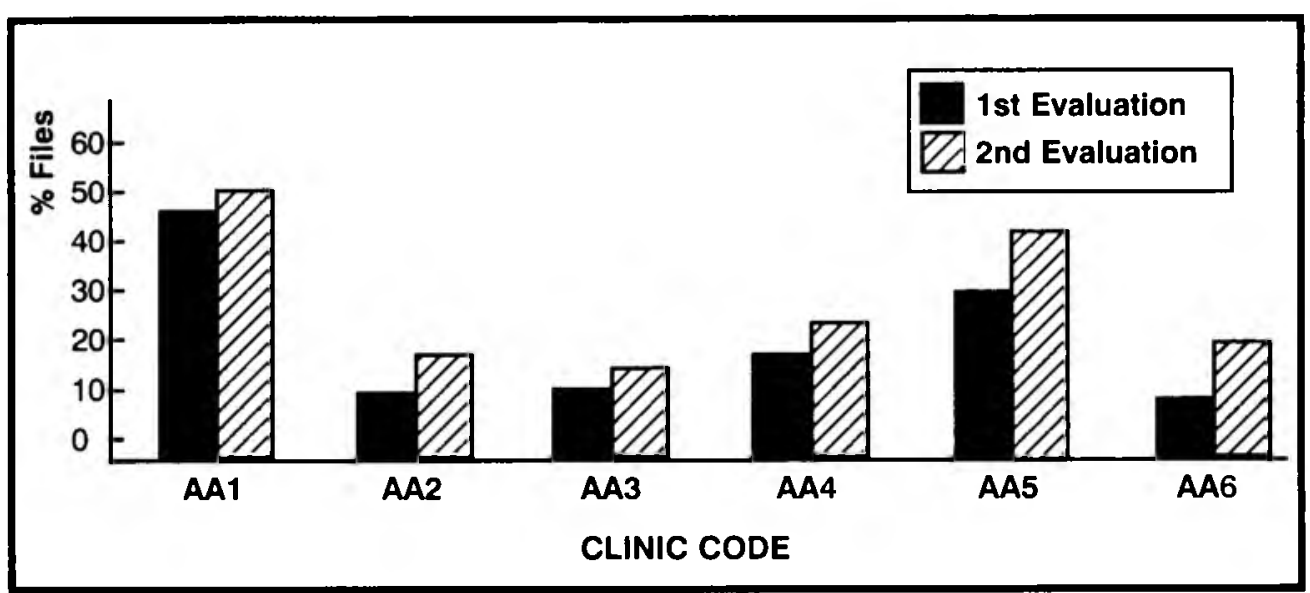

family and community. Family and client involvement in the setting of goals with health workers made the plans realistic. During this process the health professional provided the education and support the client and family needed. Nurses had to assist families to accept what could realistically be expected from the clients. This change in family perspective caused a significant improvement in the emotional climate in the family.

When looking at the results in figure 1 and figure 2, Clinic AA2; AA3; and AA4 about $20 \%$; $20 \%$; and $12 \%$ target symptoms respectively were not identified. These 3 clinics had difficulty in making frequent contacts with their clients and families due to a long distance between the clients' home and the clinic and also due to bad roads to these villages which made it difficult for nurses to conduct home visits for this exercise. Nurses had to use their own transport on weekends to achieve success. Clinic AA1 and AA6 had good results because of the following reasons :

* Clinic AA1 is a local authority clinic serving a periurban and urban area where it is easy to conduct home visits. This clinic has a partnership with the University which made it easy for nurses to get expertise and support from psychologists and social workers as they visit the clinic. Also there is a social worker attached to the clinic who gave support and advice.

* Clinic AA6 has a very 
supportive community and a health desk which provides support and at times transport nurses to various homes when requested.

In figure 3 Clinics AA1; AA2; AA3; AA4 and $A A 6$ had a difficulty in setting a plan for the family in the first 12 months. Retraining had to be done around what was available in each family and also in the community. Although there were better resources for Clinic AA1 these were not fully utilized before retraining. In the rest of the clinics participants had a difficulty in identifying scarce resources which could be used within a home. This improved after 18 months. Clinic AA5 had a good working relationship with the families involved. One of the families assisted in making plans with the other families and a team spirit developed between these families.

In figure 4 participants in Clinic AA1; AA3; AA4 and AA5 had a difficulty in deciding complete steps to achieve the goal. Most steps were appropriate but not complete which made it difficult for the families to implement. Gaps were identified by each clinic and completed. Only clinic AA6 had a similar problem after 18 months. This had no significance in the achievement of most goals.

In terms of vocational rehabilitation, it was difficult to get any job placement for clients due to high unemployment in the area. Only in one rural and one urban clinic was a vocational project run by the PHC nurses. In the other clinics the families had to start a "project" for their family member e.g. a garden.

Rehabilitation can be taught to all PHC nurses as part of their daily work to enable the clients to learn new skills, re-learn old skills that were lost due to illness and to utilize the remaining abilities. The family can be taught how to do this so that they take over the rehabilitation process under the supervision of the health worker. To complete the process, however, communities will have to be involved to start small projects from which disabled clients can learn work related skills and behaviour. These projects can also generate income for use by clients and communities.

It is recommended that social services be sensitised into the need for financial assistance of the chronic mentally ill persons so that their rehabilitation and personal needs are met. There is need for the education of the family in the correct use and objective of the disability grant. A policy should be set up in which rehabilitation of the disabled clients in the community will be included in order to guide the communities in continuing rehabilitation as a community responsibility. A multi disciplinary approach into the training of disabled persons within these rehabilitation projects must be clearly identified. Psychosocial rehabilitation in the PHC approach must be seen as part of treatment and be started at the beginning of treatment to enable clients to be fully integrated within the society.

\section{6. list of references}

Anthony, W. Cohen, M.A. \& Farkas, M. 1990. Psychiatric Rehabilitation. Boston: Centre for Psychiatric Rehabilitation.

Krauss, J.B. \& Slavinsky, A.T. 1982. The Chronically ill Psychiatric Patient and the Community,Boston. Blackwell Scientific Book Distributers.

Munich, R. L. 1993. The Boundaries of Psychiatric Rehabilitation. Hospital and Community Psychiatry, 44 (7).

Reeler, A. P. 1987 Chiweshe NurseCounsellor Programme Trainee's Manual,Department of Psychiatry and Community Medicine, University of Zimbabwe.

Sokhela, N.E. \& Uys, L.R. 1996.

The Integration of Psychiatric Component into the PHC - Unpublished study, University of Natal, Durban.

Sullivan, P. 1994. A long and winding road : The process of recovery from mental illness. Innovations and Research,3 (3).

Uys, L.R. 1991. A Theoretical Framework for Psychiatric Rehabilitation, Curationis, 14 (3) $1-5$ 